\title{
Women's First Names: A Semantic Differential Analysis*
}

\section{E. D. LAWSON}

Tres

HE EFFECTIVENESS of the semantic differential technique developed by Osgood et $a l .{ }^{1}$ for the study of men's names has been demonstrated in recent investigations. ${ }^{2}$ According to Osgood the semantic differential can measure concepts or ideas on three basic dimensions of meaning: Evaluation, Potency, and Activity, and also place them in a three-dimensional space of meaning. Locating concepts, such as names, in space has the advantage of showing how concepts relate to one another, as well as to reference points. Work with men's names has (1) confirmed the existence of stereotypes, (2) shown a tendency toward agreement in ratings by men and women, and (3) indicated a degree of relationship between frequency of occurrence of a name and its rating.

The important psychological aspects which impel the study of men's names ${ }^{3}$ should apply equally well to women's names. But while there are a number of reports on men's names, systematic studies of women's names are fewer, possibly because of the difficulties in studying women's names. Women's names shift more in popularity ${ }^{4}$ and there is a greater variety of their names.

* Appreciation is expressed to the Computer Center and the Instructional Resources Center, State University College at Fredonia and also to Betsy Weaver, Chris Barry, and Sam Peng for assistance in this project.

1 Charles E. Osgood, George J. Suci, and Percy Tannenbaum, The Measurement o Meaning (Urbana, University of Illinois Press, 1957), p. 342.

2 E. D. Lawson, "Semantic Differential Analysis of Men's First Names," Journal o Psychology, 78 (1971), pp. 229-240; E. D. Lawson, "Men's First Names, Nicknames, and Short Names: A Semantic Differential Analysis," Names, 21 :1 (March, 1973), pp. 22-27.

3 William L. Albott and James L. Bruning, "Given Names: A Neglected Social Variable," Psychological Record, 20 (1970), pp. 527-533.

Paul Plottke, "The Child and His Name," Individual Psychology Bulletin, 8 (1950), pp. 150-157.

Myron Brender, "Some Hypotheses About the Psychodynamic Significance of Infant Name Selection," Names, 11:1 (March, 1963), pp. 1-9.

- F. Warren Wright, "Fashions in Girls' Names at Smith College," Names, 2:3 (September, 1954), pp. 166-168.

New York City Bureau of Health Statistics and Analysis, as reported in New York Times, May 13, 1972, p. 16.

New York State Health Department Office of Biostatistics, as reported in Dunkirk (N.Y.) Observer, July 10, 1973. 
Are there stereotypes of women's names? If so, do men and women agree on these stereotypes? Is frequency of a name related to its attractiveness? Do a relatively few names (as with men) account for a large percentage of names chosen? This study attempts to answer these questions.

\section{Method}

The rating procedure was the same as reported earlier with the studies of men's names. ${ }^{5}$ Each respondent rated each concept on ten bipolar scales such as valuable--worthless, fast-slow, large-small. Two groups of concepts were rated: dimension concepts, and women's names. The dimension concepts Good and Bad, Strong and Weak, Active and Passive served as assumed reference points for Osgood's Evaluative, Potency, and Activity factors respectively. The names chosen were the 20 most common on the campus. For selection of names all women's names in the student directory were counted $(1,872)$. There were 364 different names even when obviously similar names (for example, Ann and Anne, Lynne and Lynn, Margaret and Margret) were combined. This compares with only 176 different names for 1,292 men.

The dimension concepts were rated first, in random order, followed by the names, also in random order. The randomization was such that no two subjects had either the dimension concepts or the names in the same order. The subjects were 50 men and 50 women undergraduates randomly selected at State University College, Fredonia.

\section{Results}

The data were analyzed using computer programs developed for use with the semantic differential. ${ }^{6}$ The first question is whether stereotypes were found. Means and standard deviations for the concepts were computed on each subscale separately for men and for women. Data inspection shows that the dimension concepts and the names are spread over a good range and that standard deviations are reasonably small. Previous experience with the semantic differential confirms that with this kind of range and standard deviations stereotypes do exist; that is, the images held by men, as a group, on the dimension concepts and on the names are shared. This is also true for women.

Before answering the question as to whether men and women agree on these stereotypes, it is first necessary to describe and discuss results with the $D$ (distance) scores. Essentially the $D$ score is a difference profile

5 Lawson, 1971, p. 231 ; 1973, p. 23.

- E. D. Lawson, George H. Golden, Jr., and Kathy J. Chmura, "Computer Programs for the Semantic Differential," Educational and Psychological Measurement, 32 (1972), pp. 779-784. 
between ratings of two concepts on the same subscales. Thus scores on the ten subscales (valuable-worthless, fast-slow, etc.) for the concept Good would represent one profile, for the concept Bad another profile. The $D$ score is the sum of the differences on the subscales and is found by the generalized distance formula $D=\sqrt{\Sigma \mathrm{d}^{2}}$ in which $d$ is the difference in rating of a concept on the same two subscales. The ten subscale scores were combined into a single $D$ score. For concepts perceived as close together, as Good and Mary, $D$ would be small; for concepts far apart, as Good and Bad, $D$ would be large. For each subject $D$ scores were computed between each concept and every other concept. The Wilcoxon matched pairs procedure was used with $D$ s to determine the level of proximity to Good, Strong, and Active (the attractive ends of each dimension), the Evaluative, Potency, and Activity for each name. All of the women's names were closer to the Good, Strong, and Active ends of the assumed continua. Wilcoxon probabilities for the dimensions were then converted into ranks and are shown in the table. In addition to the ranks for each name on the dimensions, there is a composite score determined by averaging the three dimension scores, and the frequency of occurrence of each name on the campus.

Men ranked Karen, Nancy, and Mary as most attractive (closest to Good, Strong, and Active) on the composite score. Women were somewhat different. They ranked Susan, Joan, and Jean the most attractive. Men placed Patricia, Linda, and Cynthia as less desirable; women, Cynthia, Diane, and Sharon. However, actual differences between the names were very slight. All of the names were on the attractive ends of the continua. In fact all names on the Evaluative dimension were closer to Good than Bad at a very high statistical level (.01).

A quite different way of looking at the same data is by construction of a three-dimensional model. The group means were again used, this time for $D$ s based upon the entire group. Figures 1 and 2 show models based upon group $D$ s. While the procedures are somewhat different, the interpretations generally follow the table. The models show perhaps somewhat better than the table how closely the stereotypes derived from the ratings cluster together.

Just how similar were the ratings of men and women? Correlations computed on each dimension and the composite scores between the rankings of men and women were Good-Bad, .61; Strong-Weak, -.06; Active-Passive, -.05; Composite, .21. These compare with correlations of .26, .18, .35, and .32 respectively for men's names. ${ }^{7}$ Thus on one dimension, the Evaluative (the most important according to Osgood), there is a significantly high agreement. As for the insignificant correlations on the

7 Lawson, 1971, p. 235. 


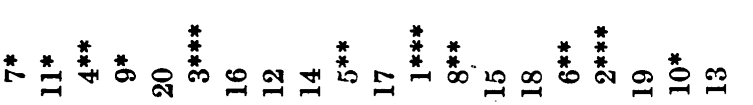
苛

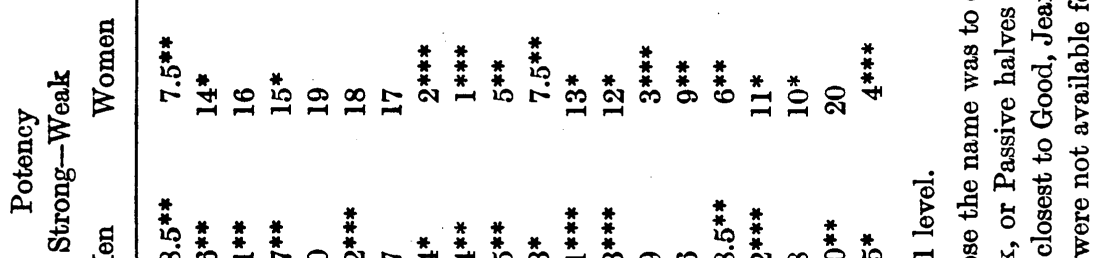

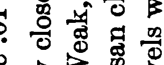
吾言范

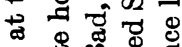

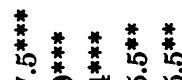

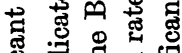

曾

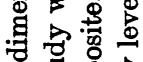

현

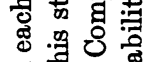
ธํㅝ융

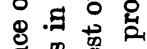
㻤 点 $\quad$ 角 . 


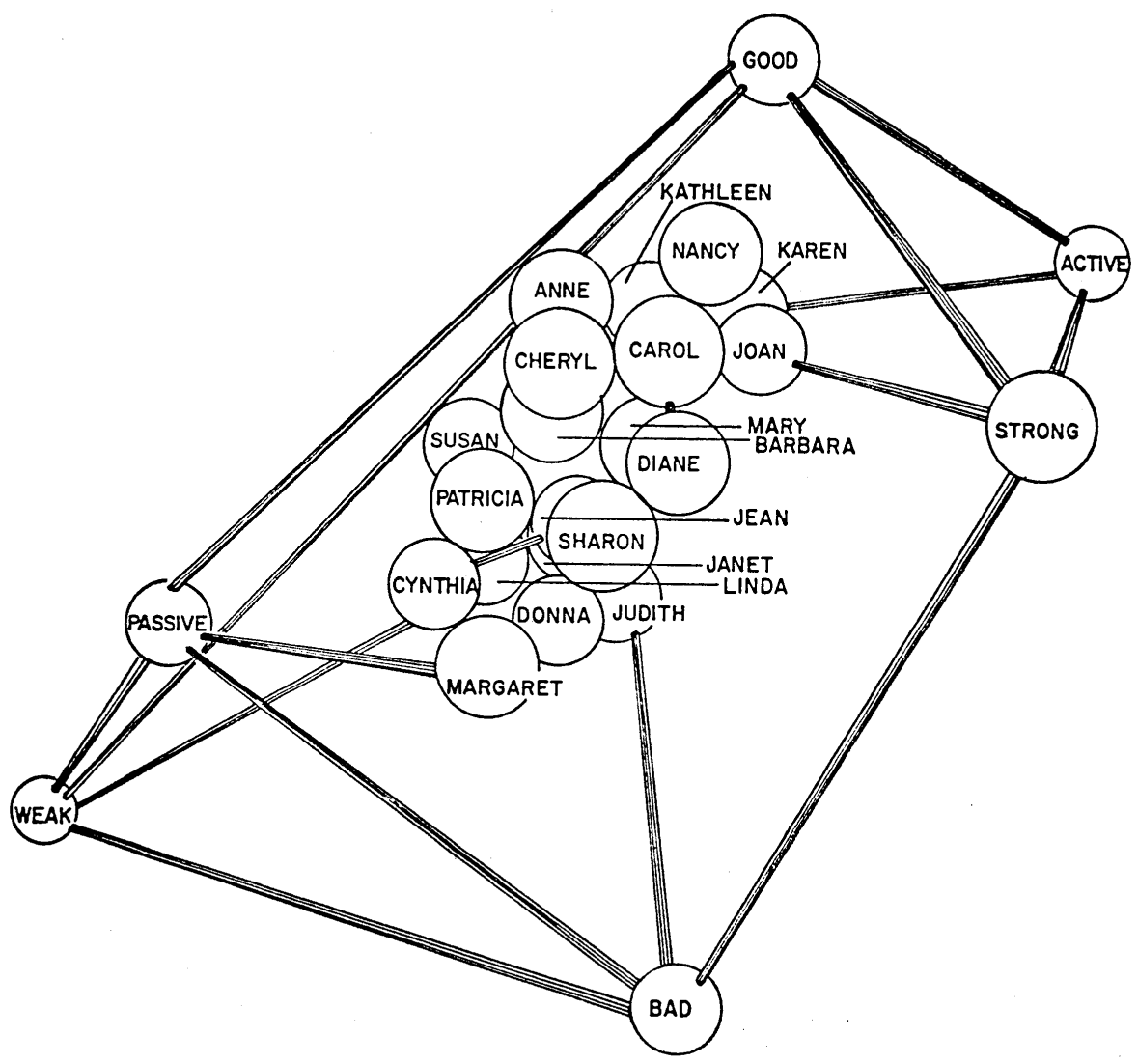

Figure 1.

Three-Dimensional Model of Men's Ratings of Women's First Names

other dimensions we can only offer the speculation that while the concepts and dimension of Good-Bad in reference to women's names mean the same to men and women, the other dimensions, Strong-Weak, and Active-Passive, carry somewhat different meanings.

Frequency of a name and its rating were also explcred. On the basis of the study of men's names there was reason to believe there might be some relationship between frequency of a name and its rating on the different dimensions. Correlations between frequency of common men's names and dimensions ranged from .16 to .68. Those with women's names were all insignificantly low, below .16. Using the 20 most common names probably lowered the correlation somewhat. There would be a statistical advantage in taking names from the whole spectrum of usage.

How were women's names distributed? Were they scattered over a larger range than those of men? There certainly was more of a range 


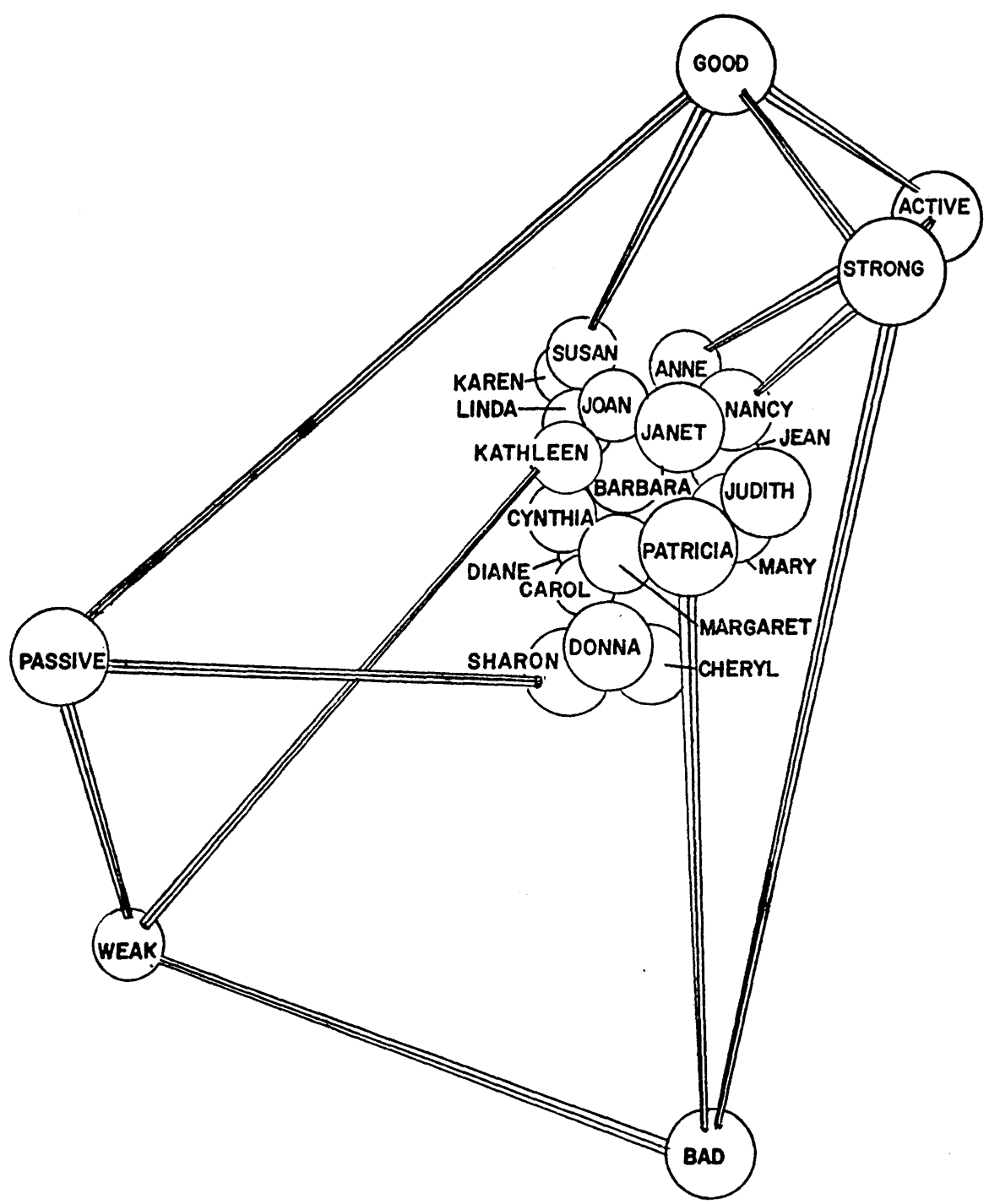

Figure 2.

Three-Dimensional Model of Women's Ratings of Women's First Names

(variety) of names for women, $364 \mathrm{vs} .176$. The next question is whether the frequencies were spread out over the whole distribution. Some might assume that, since there are more women's names, the frequencies would be spread over the whole distribution or at least skewed proportionately to men. With men, 20 names out of 176 (11 percent) accounted for 58 percent of the total number. With women, 20 names out of 364 (5.5 percent) accounted for 44 percent of the total number. A relatively small 
number of women's names accounts for a substantial percentage of the total number of names chosen. The conclusion, then, with women as with men, is that a relatively small number of names are overchosen.

Results of this investigation confirm the existence of stereotypes for common women's names. All names were rated closer to Good than Bad, to Strong than Weak, to Active than Passive. On the Evaluative dimension, all names were rated significantly close to Good. The stereotypes of men and women are significantly correlated on the Evaluative dimension but not on the others. With this limited sample frequency of a name was not found to be correlated with attractiveness. A relatively few popular names were held by a sizeable percentage of the students on the sample campus. Further studies need to include those names that are less popular as well.

The State University College at Fredonia, New York

\section{NECROLOGY}

Dr. Francis Lee Utley, Professor Emeritus of English at the Ohio State University, internationally known folklorist, and past president of the American Name Society, American Folklore Society, and College English Association, died on March 9, 1974 in Columbus, Ohio, at the age of 66. A complete obituary will appear in a forthcoming issue of Names. 